CUBO A Mathematical Journal

Vol.14, No 02, (61-80). June 2012

\title{
Bicomplex Numbers and their Elementary Functions
}

\author{
M.E. Luna-Elizarrarás, M. Shapiro \\ Departamento de Matemáticas, \\ E.S.F.M del I.P.N., México. \\ and \\ D.C. Struppa ${ }^{1}$, A. Vajiac \\ Schmid College of Science and Technology, \\ Chapman University, Orange California, \\ 1 email: struppa@chapman.edu
}

\begin{abstract}
In this paper we introduce the algebra of bicomplex numbers as a generalization of the field of complex numbers. We describe how to define elementary functions in such an algebra (polynomials, exponential functions, and trigonometric functions) as well as their inverse functions (roots, logarithms, inverse trigonometric functions). Our goal is to show that a function theory on bicomplex numbers is, in some sense, a better generalization of the theory of holomorphic functions of one variable, than the classical theory of holomorphic functions in two complex variables.
\end{abstract}

\section{RESUMEN}

En este artículo introducimos el álgebra de números bicomplejos como una generalización del campo de números complejos. Describimos cómo definir funciones elementales en tales álgebras (polinomios y funciones exponenciales y trigonométricas) así como sus funciones inversas (raíces, logaritmos, funciones trigonométricas inversas). Nuestro objetivo es mostrar que una teoría de funciones sobre números bicomplejos es, en cierto sentido, una mejor generalización de la teoría de funciones holomorfas de una variable compleja, que la teoría de funciones holomorfas en dos variables complejas.

Keywords and Phrases: Bicomplex numbers, Elementary functions

2010 AMS Mathematics Subject Classification: 30G35 


\section{Introduction}

Consider two $C^{1}$ functions $u, v$ from $\mathbb{R}^{2}=\left\{\left(x_{1}, x_{2}\right): x_{1} \in \mathbb{R}, x_{2} \in \mathbb{R}\right\}$ to $\mathbb{R}$. It is well known that if these two functions satisfy the so-called Cauchy-Riemann system

$$
\begin{gathered}
\frac{\partial u}{\partial x_{1}}=\frac{\partial v}{\partial x_{2}} \\
\frac{\partial u}{\partial x_{2}}=-\frac{\partial v}{\partial x_{1}}
\end{gathered}
$$

then the function $\mathfrak{f}\left(x_{1}+\mathfrak{i} x_{2}\right):=\mathfrak{u}\left(x_{1}, x_{2}\right)+\mathfrak{i} v\left(x_{1}, x_{2}\right)$ admits complex derivative (i.e. the limit $\lim _{h \rightarrow 0} \frac{f(z+h)-f(z)}{h}$ exists), and is what we call a holomorphic function. This observation is the key point of the theory of one complex variable, and shows that the entire theory relies on considering pairs of differentiable functions connected by a simple system of linear, constant coefficients, first order, partial differential equations.

There are different ways to attempt to generalize this observation to the case of more pairs of real variables. For example, if we consider two such pairs defined on two independent sets of variables (i.e. a map defined on $\mathbb{R}^{4}$ with values in $\mathbb{R}^{4}$ ), one can consider quaternion valued functions of a quaternionic variable, and a very interesting theory of holomorphicity was developed by Fueter [3] (though others like Moisil 44 and Mosil-Teodorescu [5] introduced similar ideas before him). Another way to generalize this observation consists in looking at maps $\vec{f}=\left(f_{1}, f_{2}\right)$ from $\mathbb{C}^{2}$ to $\mathbb{C}^{2}$, and to ask that each component $f_{1}, f_{2}$ be holomorphic in both variables in $\mathbb{C}^{2}$, without assuming any additional relationship between them. Though both generalizations are important, and give rise to large and interesting theories, we believe that there is another even more appropriate generalization, which so far has not received enough attention.

To this purpose, we propose to complexify the Cauchy-Riemann system and to apply it to pairs of holomorphic functions $u, v$ from $\mathbb{C}^{2}=\left\{\left(z_{1}, z_{2}\right): z_{1} \in \mathbb{C}, z_{2} \in \mathbb{C}\right\}$ to $\mathbb{C}$, so that the pair $(u, v)$ can be interpreted as a map of $\mathbb{C}^{2}$ to itself. It is then natural to ask whether it makes any sense to consider pairs $(u, v)$ for which the following system is satisfied:

$$
\begin{gathered}
\frac{\partial u}{\partial z_{1}}=\frac{\partial v}{\partial z_{2}} \\
\frac{\partial u}{\partial z_{2}}=-\frac{\partial v}{\partial z_{1}} .
\end{gathered}
$$

Formally, we have replaced $\mathbb{R}$ by $\mathbb{C}$, and differentiability in the real sense by holomorphicity. Does this have any implications on the pair $(u, v)$ ? As it turns out, it is possible to give a very interesting interpretation of this complexified Cauchy-Riemann system, if we endow the pair $\left(z_{1}, z_{2}\right)$ with a special algebraic structure. Instead of considering $\left(z_{1}, z_{2}\right)$ as a point in $\mathbb{C}^{2}$ we now consider, in analogy with what we did in the case of $\mathbb{R}^{2}$, a new space whose elements are of the form $\mathbf{Z}=z_{1}+\mathbf{j} z_{2}$, where $\mathbf{j}$ is a new imaginary unit (i.e. $\mathbf{j}^{2}=-1$ ), which commutes with the original imaginary unit $\mathbf{i}$. This creates a new algebra, the algebra of bicomplex numbers, and as we will 
show in the next sections, such an algebra enjoys most of the properties one would expect from a good generalization of the field of complex numbers.

There is another equally interesting way of introducing bicomplex numbers. We recall, for example, that complex numbers are important because they allow the factorization of the positively definite real quadratic form

$$
x_{1}^{2}+x_{2}^{2}=\left(x_{1}+\mathbf{i} x_{2}\right)\left(x_{1}-\mathbf{i} x_{2}\right),
$$

which defines the geometry of the plane and is the symbol of the 2-dimensional Laplace operator. One may therefore ask whether it is possible to factor the complex analog of the form, i.e. the $\mathbb{C}$-valued quadratic form $z_{1}^{2}+z_{2}^{2}$, which is the symbol of the 2-dimensional complex Laplace operator. A trivial answer is to express such form as a product of two linear complex valued factors as

$$
z_{1}^{2}+z_{2}^{2}=\left(z_{1}+\mathbf{i} z_{2}\right)\left(z_{1}-\mathbf{i} z_{2}\right)
$$

These two factorizations may appear superficially similar, but in fact there is a deep difference between them. The factorization of $x_{1}^{2}+x_{2}^{2}$ is realized through real 2-dimensional factors, while the factorization of $z_{1}^{2}+z_{2}^{2}$ is realized through complex 1-dimensional factors. One may therefore ask whether it is possible to factor the complex quadratic form through two factors which have complex dimension 2. In order to do so, we consider a distributive, and associative (but not necessarily commutative) complex algebra over $\mathbb{C}^{2}$, and we assume we have two elements $\mathrm{a}, \mathrm{b}$ in this algebra, such that

$$
z_{1}^{2}+z_{2}^{2}=\left(z_{1}+\mathrm{a} z_{2}\right)\left(z_{1}+\mathrm{b} z_{2}\right)=z_{1}^{2}+\mathrm{a} z_{2} z_{1}+z_{1} \mathrm{~b} z_{2}+\mathrm{a} z_{2} \mathrm{~b} z_{2}
$$

This implies immediately that, for every $z_{1}, z_{2}$ we must have

$$
\mathrm{a} z_{2} z_{1}+z_{1} \mathrm{~b} z_{2}=0
$$

which, for $z_{1}=1$, gives

$$
\mathrm{a} z_{2}+\mathrm{b} z_{2}=0
$$

and therefore $a=-b$. By substituting in (1.2) we obtain

$$
\mathrm{a} z_{2} z_{1}-z_{1} \mathrm{a} z_{2}=0
$$

which, for $z_{2}=1$, gives

$$
\mathrm{a} z_{1}=z_{1} \mathrm{a} .
$$

This shows that $a$ is not a complex number, but it commutes with every complex number. By inserting these results in (1.1) we obtain

$$
z_{1}^{2}+z_{2}^{2}=z_{1}^{2}-a^{2} z_{2}^{2}
$$

i.e. $a^{2}=-1$. Once again, we have arrived to a new structure, which requires a to be a second imaginary unit, which we will call $\mathbf{j}$ in the sequel, that commutes with the initial imaginary unit $\mathbf{i}$. 
The algebra which one obtains is the bicomplex algebra. In this paper we show how to introduce elementary functions, such as polynomials, exponentials, trigonometric functions, in this algebra, as well as their inverses (something that, incidentally, is not possible in the case of quaternions). We will show how these elementary functions enjoy properties that are very similar to those enjoyed by their complex counterparts. In addition, as we will indicate below, this algebraic structure will allow us to show that any pair of holomorphic functions that satisfies the complexified CauchyRiemann system admits derivative in the sense of bicomplex numbers.

\section{The bicomplex numbers}

We gave, in the introduction, a couple of justifications for the introduction of the notion of bicomplex numbers. It is however also possible to arrive to bicomplex numbers by means of purely algebraic considerations. For example, if in a complex number $\mathbf{a}+\mathbf{i} b$ we replace the real numbers $\mathrm{a}$ and $\mathrm{b}$ by complex numbers $z_{1}=\mathrm{a}_{1}+\mathbf{i} \mathrm{a}_{2}$ and $z_{2}=\mathrm{b}_{1}+\mathbf{i} \mathrm{b}_{2}$, then we get just another complex number:

$$
z_{1}+\mathbf{i} z_{2}=\left(a_{1}+\mathbf{i} a_{2}\right)+\mathbf{i}\left(b_{1}+\mathbf{i} b_{2}\right)=\left(a_{1}-b_{2}\right)+\mathbf{i}\left(a_{2}+b_{1}\right) .
$$

If we want to obtain a new type of number, then we must use another imaginary unit, say $\mathbf{j}$, with $\mathbf{j}^{2}=-1$, and set

$$
z_{1}+\mathbf{j} z_{2}=\left(a_{1}+\mathbf{i} a_{2}\right)+\mathbf{j}\left(b_{1}+\mathbf{i} b_{2}\right),
$$

which gives a new object, outside the field of complex numbers.

If we want to be able to operate with these new numbers, we need to define the product of the two imaginary units. This was a problem that was solved by Hamilton by requiring that they anticommute, and his solution led to the introduction of quaternions. Hamilton's decision was influenced by many considerations, including the desire to obtain a field, which of course the quaternions form (a skew field). But one could explore what happens if we assume that the two new imaginary units commute. In this case we obtain a new, and lesser known object, the algebra of bicomplex numbers.

The set $\mathbb{B C}$ of bicomplex numbers is therefore defined as follows:

$$
\mathbb{B C}=\left\{z_{1}+\mathbf{j} z_{2} \mid z_{1}, z_{2} \in \mathbb{C}\right\},
$$

where $\mathbf{i}$ and $\mathbf{j}$ are commuting imaginary units, i.e. $\mathbf{i j}=\mathbf{j} \mathbf{i}, \mathbf{i}^{2}=\mathbf{j}^{2}=-1$, and $\mathbb{C}$ is the set of complex numbers with the imaginary unit $\mathbf{i}$. Thus bicomplex numbers are "complex numbers with complex coefficients", which explains the name of bicomplex, and in what follows we will try to emphazise the deep similarities between the properties of complex and bicomplex numbers. We should probably point out that bicomplex numbers were apparently first introduced in 1892 by Segre, [12], that the origin of their function theory is due to the Italian school of Scorza-Dragoni ([13], [14, [15]), and that a first theory of differentiability in $\mathbb{B} \mathbb{C}$ was developed by Price in [7]. 
Subsequently, other authors have developed further the study of these objects, [2], [6, [8], 9] . A key role, in this evolution, has been played by John Ryan, who was probably the first to understand the importance of complex Clifford Algebras (of which $\mathbb{B C}$ is the simplest example, and the only commutative one), and to highlight their role in analysis, [10, 11].

A bicomplex number can be written in cartesian form as $\mathbf{Z}=z_{1}+\mathbf{j} z_{2}$ or, at least as long as $z_{1}^{2}+z_{2}^{2} \neq 0$, in trigonometric form as

$$
\begin{aligned}
\mathrm{Z}=z_{1}+\mathbf{j} z_{2} & =\sqrt{z_{1}^{2}+z_{2}^{2}}\left(\frac{z_{1}}{\sqrt{z_{1}^{2}+z_{2}^{2}}}+\mathbf{j} \frac{z_{2}}{\sqrt{z_{1}^{2}+z_{2}^{2}}}\right) \\
& =\sqrt{z_{1}^{2}+z_{2}^{2}}(\cos \theta+\mathbf{j} \sin \theta),
\end{aligned}
$$

where the complex number $\theta$ is a solution of the system

$$
\cos (\theta)=\frac{z_{1}}{\sqrt{z_{1}^{2}+z_{2}^{2}}}, \quad \sin (\theta)=\frac{z_{2}}{\sqrt{z_{1}^{2}+z_{2}^{2}}} .
$$

Since for the particular case of real $z_{1}$ and $z_{2}, \sqrt{z_{1}^{2}+z_{2}^{2}}$ is the modulus of a complex number and $\theta$ is its argument, the complex number $\sqrt{z_{1}^{2}+z_{2}^{2}}$ is called the complex modulus of the bicomplex number $\mathbf{Z}$, denoted by $|Z|_{c}$, and $\theta$ is called the complex argument of $Z$, denoted by $\arg _{c}(Z)$. These names can be given a deeper justification, but this is beyond the scope of this article.

It can be shown by elementary complex analysis that the apparent ambiguities in formula (2.1) can always be resolved by choosing either the principal or the secondary branch of the complex square root $\sqrt{z_{1}^{2}+z_{2}^{2}}$. Either way formula (2.1) is well-defined.

Now the addition and the multiplication of bicomplex numbers are introduced in a natural way: given $Z_{1}=z_{11}+\mathbf{j} z_{12}$ and $Z_{2}=z_{21}+\mathbf{j} z_{22}$ in $\mathbb{B C}$, then

$$
Z_{1}+Z_{2}:=\left(z_{11}+z_{21}\right)+\mathbf{j}\left(z_{12}+z_{22}\right)
$$

and

$$
Z_{1} \cdot Z_{2}:=\left(z_{11}+\mathbf{j} z_{12}\right)\left(z_{21}+\mathbf{j} z_{22}\right)=\left(z_{11} z_{21}-z_{12} z_{22}\right)+\mathbf{j}\left(z_{11} z_{22}+z_{21} z_{12}\right) .
$$

It is a simple exercise left to the reader to verify the following

Proposition 1. $(\mathbb{B C},+, \cdot)$ is a commutative ring, i.e.

(1) The addition is associative, commutative, with identity element $\mathbf{0}=\mathbf{0}+\mathbf{j} \mathbf{0}$, and all bicomplex numbers have an additive inverse. This is to say that $(\mathbb{B C},+)$ is an Abelian group.

(2) The multiplication is associative, commutative, with identity element $\mathbf{1}=1+\mathbf{j} 0$. 
(3) The multiplication is distributive with respect to the addition, i.e. for any $Z, Z_{1}, Z_{2} \in \mathbb{B C}$, we have:

$$
\mathrm{Z}\left(\mathrm{Z}_{1}+\mathrm{Z}_{2}\right)=\mathrm{ZZ}_{1}+\mathrm{ZZ}_{2}
$$

Remark 2. The process described above has allowed us to endow the complex linear space $\mathbb{C}^{2}$ with a structure of a commutative complex algebra. Note that quaternions form only a real, not a complex algebra. On the other hand, quaternions are a (skew) field, while we will soon show that not all bicomplex numbers have a multiplicative inverse.

\subsection{Bicomplex Conjugation}

Given a bicomplex number $\mathbf{Z}=z_{1}+\mathbf{j} z_{2}$, its (bicomplex) conjugate is defined by

$$
\mathrm{Z}^{\dagger}:=z_{1}-\mathbf{j} z_{2}
$$

We immediately notice that

$$
\mathrm{Z} \cdot \mathrm{Z}^{\dagger}=z_{1}^{2}+z_{2}^{2} \in \mathbb{C} .
$$

This last equality is only apparently similar to the corresponding identity for complex numbers; however the quadratic form in (2.6) takes complex values (rather than real non-negative values), and this implicates significant differences with the complex situation. In particular it implies that a bicomplex number $\mathbf{Z}=z_{1}+\mathbf{j} z_{2}$ is invertible if and only if

$$
\mathrm{Z} \cdot \mathrm{Z}^{\dagger}=z_{1}^{2}+z_{2}^{2} \neq 0
$$

In this case, it is easy to verify that the inverse of $Z$ is given by

$$
Z^{-1}=\frac{Z^{\dagger}}{z_{1}^{2}+z_{2}^{2}}
$$

If both $z_{1}$ and $z_{2}$ are non-zero but the sum $z_{1}^{2}+z_{2}^{2}=0$, then the corresponding bicomplex number $Z=z_{1}+\mathbf{j} z_{2}$ is a zero divisor. In fact all zero divisors $Z=z_{1}+\mathbf{j} z_{2}$ in $\mathbb{B C}$ are characterized by the equations $z_{1}^{2}=-z_{2}^{2}$, i.e. $z_{1}= \pm \mathbf{i} z_{2}$. Thus all zero divisors are of the form:

$$
\mathrm{Z}=\lambda(1 \pm \mathbf{i j})
$$

for any $\lambda \in \mathbb{C} \backslash\{0\}$.

The following proposition is a simple exercise.

Proposition 3. The bicomplex numbers

$$
\mathbf{e}:=\frac{1+\mathbf{i j}}{2} \quad \text { and } \quad \mathbf{e}^{\dagger}:=\frac{1-\mathbf{i j}}{2}
$$


are zero divisors, which are linearly independent in the $\mathbb{C}$-linear space $\mathbb{C}^{2}$, and satisfy the identities:

$$
\begin{aligned}
& \mathbf{e}+\mathbf{e}^{\dagger}=1, \quad \mathbf{e}-\mathbf{e}^{\dagger}=\mathbf{i j}, \\
& \mathbf{e} \cdot \mathbf{e}^{\dagger}=0, \quad \mathbf{e}^{2}=\mathbf{e}, \quad \mathbf{e}^{\dagger^{2}}=\mathbf{e}^{\dagger} .
\end{aligned}
$$

The next property has no analog for complex numbers, and it exemplifies one of the interesting peculiarities of the bicomplex setting. For any bicomplex number $Z=z_{1}+\mathbf{j} z_{2} \in \mathbb{B C}$ one can write

$$
\mathbf{Z}=\alpha \mathbf{e}+\beta \mathbf{e}^{\dagger},
$$

where $\alpha=z_{1}-\mathbf{i} z_{2}$ and $\beta=z_{1}+\mathbf{i} z_{2}$ are uniquely defined complex numbers. Formula (2.8) is called the idempotent representation of $\mathbf{Z}$.

This shows that the set $\left\{\mathbf{e}, \mathbf{e}^{\dagger}\right\}$ is another basis for the complex space $\mathbb{B C}$, and writing $Z$ as a pair $\left(z_{1}, z_{2}\right)$ in $\mathbb{C}^{2}$, one has the transition formula

$$
\left(\begin{array}{c}
z_{1} \\
z_{2}
\end{array}\right)=\left(\begin{array}{cc}
\frac{1}{2} & \frac{1}{2} \\
-\frac{1}{2 \mathbf{i}} & \frac{1}{2 \mathbf{i}}
\end{array}\right)\left(\begin{array}{l}
\alpha \\
\beta
\end{array}\right) .
$$

Note that this new basis is orthogonal with respect to the Euclidean inner product in $\mathbb{C}^{2}$ which is given for $\left(z_{1}, w_{1}\right)$ and $\left(z_{2}, w_{2}\right)$ by

$$
\left\langle\left(z_{1}, w_{1}\right),\left(z_{2}, w_{2}\right)\right\rangle_{\mathbb{C}^{2}}:=z_{1} \overline{z_{2}}+w_{1} \overline{w_{2}} .
$$

Since $\mathbf{e}=\left(\frac{1}{2}, \frac{\mathbf{i}}{2}\right)$ and $\mathbf{e}^{\dagger}=\left(\frac{1}{2},-\frac{\mathbf{i}}{2}\right)$ in $\mathbb{C}^{2}$, we have $\left\langle\mathbf{e}, \mathbf{e}^{\dagger}\right\rangle_{\mathbb{C}^{2}}=0$, and

$$
\langle\mathbf{e}, \mathbf{e}\rangle_{\mathbb{C}^{2}}=\left\langle\mathbf{e}^{\dagger}, \mathbf{e}^{\dagger}\right\rangle_{\mathbb{C}^{2}}=\frac{1}{2},
$$

so that we have an orthogonal but not orthonormal basis for $\mathbb{C}^{2}$.

The following result shows the importance of the idempotent representation of bicomplex numbers in all algebraic operations.

Proposition 4. The addition and multiplication of bicomplex numbers can be realized "term-byterm" in the idempotent representation. Specifically, if $Z_{1}=\alpha \mathbf{e}+\beta \mathbf{e}^{\dagger}$ and $Z_{2}=\gamma \mathbf{e}+\delta \mathbf{e}^{\dagger}$ are two bicomplex numbers, then

$$
\begin{aligned}
Z_{1}+Z_{2} & =(\alpha+\gamma) \mathbf{e}+(\beta+\delta) \mathbf{e}^{\dagger}, \\
Z_{1} \cdot Z_{2} & =\alpha \gamma \mathbf{e}+\beta \delta \mathbf{e}^{\dagger}, \\
Z_{1}^{n} & =\alpha^{n} \mathbf{e}+\beta^{n} \mathbf{e}^{\dagger} .
\end{aligned}
$$


Moreover, the inverse of an invertible bicomplex number $\mathbf{Z}=\alpha \mathbf{e}+\beta \mathbf{e}^{\dagger}$ is given by

$$
\mathbf{Z}^{-1}=\alpha^{-1} \mathbf{e}+\beta^{-1} \mathbf{e}^{\dagger}
$$

where $\alpha^{-1}$ and $\beta^{-1}$ are the complex multiplicative inverses of $\alpha$ and $\beta$, respectively.

\section{Bicomplex Derivatives}

\section{1}

Let $f: U \subset \mathbb{B C} \rightarrow \mathbb{B C}$ be a bicomplex function. There is a definition for the derivative of a bicomplex function (see e.g. 7]) which looks quite similar to its complex counterpart.

Definition 5. The derivative of the function $f$ at a point $Z_{0} \in U$ is the limit, if it exists,

$$
f^{\prime}\left(Z_{0}\right):=\lim _{Z \rightarrow Z_{0}} \frac{f(Z)-f\left(Z_{0}\right)}{Z-Z_{0}}
$$

for $Z$ in the domain of $f$ such that $Z-Z_{0}$ is an invertible bicomplex number.

It is never emphasized in the literature that this limit is tacitly taken in the usual Euclidean topology of $\mathbb{C}^{2}$ (we call this the usual Euclidean convergence in $\mathbb{B C}$ ), which seems not to be the one generated by the natural structure of $\mathbb{B C}$ (see for instance (2.7)). We will show below that there is another, equivalent approach to the limit of a bicomplex function which employs the specific algebraic structure of bicomplex numbers.

\section{2}

Let $Z_{n}=\alpha_{n} \mathbf{e}+\beta_{n} \mathbf{e}^{\dagger}$ for $n \geq 1$, be a sequence of bicomplex numbers.

Definition 6 . The sequence $\left\{Z_{n}\right\}_{n \geq 1}$ is said to converge component-wise if the sequences of complex numbers $\left\{\alpha_{n}\right\}$ and $\left\{\beta_{n}\right\}$ are convergent in the complex plane to complex numbers $\alpha_{0}$ and $\beta_{0}$. We then write that $Z_{n} \rightarrow Z_{0}:=\alpha_{0} \mathbf{e}+\beta_{0} \mathbf{e}^{\dagger}$, and we say that $Z_{n}$ has limit $Z_{0}$.

Formula (2.9) shows the equivalence between the usual Euclidean convergence and the componentwise convergence defined above. Moreover, the Euclidean convergence is the general definition of convergence in $\mathbb{C}^{2}$, but the component-wise convergence expresses the relation between the topology and the algebraic structure of $\mathbb{B C}$.

\section{3}

Component-wise convergence of sequences allows us to define the notion of component-wise limits of bicomplex functions. 
Consider a bicomplex function $f=g+j h, f: U \subset \mathbb{B C} \rightarrow \mathbb{B C}$, and its idempotent representation $f=u \mathbf{e}+v \mathbf{e}^{\dagger}$. If for any sequence $\left\{Z_{n}\right\}_{n} \geq 1$ component-wise convergent to $Z_{0}$, the complex number sequences $\left\{\mathfrak{u}\left(Z_{n}\right)\right\}$ and $\left\{v\left(Z_{n}\right)\right\}$ are convergent in $\mathbb{C}$ to $\lambda$ and $\mu$ respectively, then the function $\boldsymbol{f}$ has a (usual) limit as $\mathbf{Z} \rightarrow \mathbf{Z}_{0}$ (with respect to the canonical topology in $\mathbb{C}^{2}$ ), and we have:

$$
\lim _{Z \rightarrow Z_{0}} f(Z)=\lambda \mathbf{e}+\mu \mathbf{e}^{\dagger} .
$$

A description of continuity of a bicomplex function which is compatible with the algebraic structure of $\mathbb{B C}$ follows immediately.

We mentioned in the introduction that bicomplex numbers are the appropriate setting to consider a complexification of the Cauchy-Riemann equations. That this is the case is demonstrated by the following important result:

Theorem 7 . Let $U$ be an open set in $\mathbb{B C}$, whose variable we indicate with $Z=z_{1}+\mathbf{j} z_{2}$ and let $f: U \rightarrow \mathbb{B C}$ be such that $f=u+j v \in C^{1}(U)$. Then $f$ admits bicomplex derivative $f^{\prime}$ if and only if:

(1) $u$ and $v$ are complex holomorphic in $z_{1}$ and $z_{2}$

(2) $\frac{\partial u}{\partial z_{1}}=\frac{\partial v}{\partial z_{2}}$ and $\frac{\partial u}{\partial z_{2}}=-\frac{\partial v}{\partial z_{1}}$ on $\mathrm{u}$.

\section{Bicomplex polynomials}

Let

$$
p(Z)=\sum_{k=0}^{n} A_{k} Z^{k}
$$

be a bicomplex polynomial of degree $\mathrm{n}$, with $\mathbf{Z}=z_{1}+\mathbf{j} z_{2}=\alpha \mathbf{e}+\beta \mathbf{e}^{\dagger}$, and bicomplex coefficients $A_{k}=\gamma_{k} \mathbf{e}+\delta_{k} \mathbf{e}^{\dagger}$, for $k=0 \ldots n$. Then $Z^{k}=\alpha^{k} \mathbf{e}+\beta^{k} \mathbf{e}^{\dagger}$ and we can rewrite $p(Z)$ as

$$
p(Z)=\sum_{k=0}^{n}\left(\gamma_{k} \alpha^{k}\right) \mathbf{e}+\sum_{k=0}^{n}\left(\delta_{k} \beta^{k}\right) \mathbf{e}^{\dagger}=: \phi(\alpha) \mathbf{e}+\psi(\beta) \mathbf{e}^{\dagger} .
$$

If we denote the set of distinct roots of $\phi$ and $\psi$ by $\mathcal{S}_{1}$ and $\mathcal{S}_{2}$, and if we denote by $\mathcal{S}$ the set of distinct roots of the polynomial $p$, it is easy to see that

$$
\mathcal{S}=\mathcal{S}_{1} \mathbf{e}+\mathcal{S}_{2} \mathbf{e}^{\dagger}
$$

so that the structure of the zero-set of a bicomplex polynomial $p(Z)$ of degree $n$ is fully described by the following three cases:

(1) If both polynomials $\phi$ and $\psi$ are of degree at least one, and if $\mathcal{S}_{1}=\left\{\alpha_{1}, \ldots, \alpha_{k}\right\}$ and $\mathcal{S}_{2}=$ $\left\{\beta_{1}, \ldots, \beta_{\ell}\right\}$, then the set of distinct roots of $p$ is given by

$$
\mathcal{S}=\left\{Z_{s, t}=\alpha_{s} \mathbf{e}+\beta_{t} \mathbf{e}^{\dagger} \mid s=1, \ldots, k, t=1, \ldots, \ell\right\} .
$$


(2) If $\phi \equiv 0$, then $\mathcal{S}_{1}=\mathbb{C}$ and $\mathcal{S}_{2}=\left\{\beta_{1}, \ldots, \beta_{\ell}\right\}$, with $\ell \leq n$. Then

$$
\mathcal{S}=\left\{Z_{\mathrm{t}}=\lambda \mathbf{e}+\beta_{\mathrm{t}} \mathbf{e}^{\dagger} \mid \lambda \in \mathbb{C}, \mathrm{t}=1, \ldots, \ell\right\} .
$$

Similarly, if $\psi \equiv 0$, then $\mathcal{S}_{2}=\mathbb{C}$ and $\mathcal{S}_{1}=\left\{\alpha_{1}, \ldots, \alpha_{k}\right\}$, where $\mathrm{k} \leq \mathrm{n}$. Then

$$
\mathcal{S}=\left\{Z_{\mathrm{s}}=\alpha_{\mathrm{s}} \mathbf{e}+\lambda \mathbf{e}^{\dagger} \mid \lambda \in \mathbb{C}, \mathrm{s}=1, \ldots, \mathrm{k}\right\} .
$$

(3) If all the coefficients $A_{k}$ with the exception of $A_{0}=\gamma_{0} \mathbf{e}+\delta_{0} \mathbf{e}^{\dagger}$ are complex multiples of $\mathbf{e}$ (respectively of $\mathbf{e}^{\dagger}$ ), but $A_{0}$ has $\delta_{0} \neq 0$ (respectively $\gamma_{0} \neq 0$ ), then $p$ has no roots.

We now discuss a few examples, to give a flavor for computations in $\mathbb{B C}$. First, consider the polynomial

$$
\begin{aligned}
p(Z) & =\left(\frac{1}{2}+\mathbf{j} \frac{\mathbf{i}}{2}\right) Z^{5}+(-(1+4 \mathbf{i})+2 \mathbf{j}(2-\mathbf{i})) Z^{4}+((-11+6 \mathbf{i})-\mathbf{j}(12+11 \mathbf{i})) Z^{3} \\
& +\left(\left(\frac{29}{2}+13 \mathbf{i}\right)+\mathbf{j}\left(-13+\frac{47}{2} \mathbf{i}\right)\right) Z^{2}+\left(\left(\frac{13}{2}-17 \mathbf{i}\right)+\mathbf{j}\left(17+\frac{13}{2} \mathbf{i}\right)\right) \mathbf{Z} \\
& -\left(\frac{11}{2}+\mathbf{i}\right)+\mathbf{j}\left(1-\frac{11}{2} \mathbf{i}\right) .
\end{aligned}
$$

The corresponding complex polynomials are:

$$
\begin{aligned}
& \phi(\alpha)=\alpha^{5}-(3+8 \mathbf{i}) \alpha^{4}+2(-11+9 \mathbf{i}) \alpha^{3}+2(19+13 \mathbf{i}) \alpha^{2}+(13-34 \mathbf{i}) \alpha-(11+2 \mathbf{i}), \\
& \psi(\beta)=\beta^{4}-6 \mathbf{i} \beta^{3}-9 \beta^{2} .
\end{aligned}
$$

Their distinct roots are $\mathcal{S}_{1}=\{\mathbf{i}, 1+2 \mathbf{i}\}$ and $\mathcal{S}_{2}=\{0,3 \mathbf{i}\}$. Then $p$ has the following four roots:

$$
\mathcal{S}=\left\{\frac{1}{2} \mathbf{i}-\frac{1}{2} \mathbf{j}, 2 \mathbf{i}+\mathbf{j}, \frac{1+2 \mathbf{i}}{2}+\mathbf{j} \frac{-2+\mathbf{i}}{2}, \frac{1+5 \mathbf{i}}{2}+\mathbf{j} \frac{1+\mathbf{i}}{2}\right\} .
$$

As another example, consider the polynomial

$$
p(Z)=(1+\mathbf{j i}) Z^{2}-(\mathbf{i}-\mathbf{j}) .
$$

The associated complex polynomials are:

$$
\phi(\alpha)=2\left(\alpha^{2}-\mathbf{i}\right), \quad \psi(\beta) \equiv 0 .
$$

The null set of $p$ is

$$
\mathcal{S}=\left\{ \pm\left(\frac{\sqrt{2}}{2}+\mathbf{i} \frac{\sqrt{2}}{2}\right) \mathbf{e}+\lambda \mathbf{e}^{\dagger} \mid \lambda \in \mathbb{C}\right\}
$$

Slightly adjusting the previous example, i.e. taking $\psi(\beta) \equiv 2$, we get the polynomial

$$
p(Z)=(1+\mathbf{j i}) Z^{2}+(1-\mathbf{i})+\mathbf{j}(1-\mathbf{i}),
$$


which has no roots.

It is also important to note that a bicomplex polynomial may not have a unique factorization into linear polynomials. For example, the polynomial $p(Z)=Z^{3}-1$ has 9 solutions. Indeed, the associated complex polynomials are

$$
\phi(\alpha)=\alpha^{3}-1, \quad \phi(\beta)=\beta^{3}-1 .
$$

The set of zeros of $\phi$ and $\psi$ are, respectively:

$$
\begin{aligned}
& \mathcal{S}_{1}=\left\{\alpha_{1}=1, \alpha_{2}=-\frac{1}{2}+\mathrm{i} \frac{\sqrt{3}}{2}, \alpha_{3}=-\frac{1}{2}-\mathrm{i} \frac{\sqrt{3}}{2}\right\} \\
& \mathcal{S}_{2}=\left\{\beta_{1}=1, \beta_{2}=-\frac{1}{2}+\mathrm{i} \frac{\sqrt{3}}{2}, \beta_{3}=-\frac{1}{2}-\mathrm{i} \frac{\sqrt{3}}{2}\right\}
\end{aligned}
$$

Then the set of solutions of $p$ is

$$
\mathcal{S}=\left\{Z_{k l}=\alpha_{k} \mathbf{e}+\beta_{\ell} \mathbf{e}^{\dagger} \mid k, \ell=1 \ldots 3\right\},
$$

and we have at least two distinct factorizations:

$$
Z^{3}-1=(Z-1)\left(Z+\frac{1}{2}-\frac{\sqrt{3}}{2} \mathbf{i}\right)\left(Z+\frac{1}{2}+\frac{\sqrt{3}}{2} \mathbf{i}\right)
$$

and

$$
\mathrm{Z}^{3}-1=(\mathrm{Z}-1)\left(\mathrm{Z}+\frac{1}{2}-\mathrm{j} \frac{\sqrt{3}}{2}\right)\left(\mathrm{Z}+\frac{1}{2}+\mathrm{j} \frac{\sqrt{3}}{2}\right)
$$

It is therefore clear from what we have indicated that bicomplex polynomials do not satisfy the Fundamental Theorem of Algebra in its original form. At the same time, the following is true and summarizes the comments above.

Theorem 8 (Analogue of the Fundamental Theorem of Algebra for bicomplex polynomials). Consider a bicomplex polynomial $p(Z)=\sum_{k=0}^{n} A_{k} Z^{k}$. If all the coefficients $A_{k}$ with the exception of the free term $A_{0}=\gamma_{0} \mathbf{e}+\delta_{0} \mathbf{e}^{\dagger}$ are complex multiple of $\mathbf{e}$ (respectively of $\mathbf{e}^{\dagger}$ ), but $A_{0}$ has $\delta_{0} \neq 0$ (respectively $\gamma_{0} \neq 0$ ), then $p$ has no roots. In all other cases, $p$ has at least one root.

Corollary 9. Assume that a bicomplex polynomial $p$ of degree $n \geq 1$ has at least one root. Then:

(1) If at least one of the coefficients $A_{k}$, for $k=1 \ldots n$, is invertible, then $p$ has at most $n^{2}$ distinct roots.

(2) If all coefficients are complex multiples of $\mathbf{e}$ (respectively $\mathbf{e}^{\dagger}$ ) then $p$ has infinitely many roots.

Note that zeros of bicomplex polynomials were originally investigated in $[6]$. 


\section{The exponential function in bicomplex numbers}

In this section, we are going to introduce the exponential function of a bicomplex variable. Our approach is based on the following theorem, whose proof requires the usage of minimal mathematical tools.

Theorem 10. Let $\mathbf{Z}=z_{1}+\mathbf{j} z_{2}$ be any bicomplex number. Then the sequence

$$
Z_{n}:=\left(1+\frac{Z}{n}\right)^{n}
$$

is convergent.

Proof. The computation below proves that the sequence is component-wise convergent. Set as before $\mathbf{Z}=\alpha \mathbf{e}+\beta \mathbf{e}^{\dagger}$. Then

$$
\begin{aligned}
\left(1+\frac{Z}{n}\right)^{n} & =\left(1+\frac{\alpha}{n} \mathbf{e}+\frac{\beta}{n} \mathbf{e}^{\dagger}\right)^{n}=\left(\mathbf{e}+\mathbf{e}^{\dagger}+\frac{\alpha}{n} \mathbf{e}+\frac{\beta}{n} \mathbf{e}^{\dagger}\right)^{n} \\
& =\left(\left(1+\frac{\alpha}{n}\right) \mathbf{e}+\left(1+\frac{\beta}{n}\right) \mathbf{e}^{\dagger}\right)^{n}=\left(1+\frac{\alpha}{n}\right)^{n} \mathbf{e}+\left(1+\frac{\beta}{n}\right)^{n} \mathbf{e}^{\dagger} .
\end{aligned}
$$

By taking the limit as $\mathrm{n} \rightarrow \infty$, and relying on the fact that the corresponding sequences of complex numbers $\left(1+\frac{\alpha}{n}\right)^{n}$ and $\left(1+\frac{\beta}{n}\right)^{n}$ are convergent to the complex exponentials $e^{\alpha}$ and $e^{\beta}$, respectively, we get that the limit of the right-hand-side exists and

$$
\begin{aligned}
\lim _{n \rightarrow \infty}\left(1+\frac{Z}{n}\right)^{n} & =\lim _{n \rightarrow \infty}\left(\left(1+\frac{\alpha}{n}\right)^{n} \mathbf{e}+\left(1+\frac{\beta}{n}\right)^{n} \mathbf{e}^{\dagger}\right)=e^{\alpha} \mathbf{e}+e^{\beta} \mathbf{e}^{\dagger} \\
& =\frac{1}{2}\left(e^{\alpha}+e^{\beta}\right)+\mathbf{j} \frac{\mathbf{i}}{2}\left(e^{\alpha}-e^{\beta}\right) \\
& =\frac{1}{2}\left(e^{z_{1}-\mathbf{i} z_{2}}+e^{z_{1}+\mathbf{i} z_{2}}\right)+\mathbf{j} \frac{\mathbf{i}}{2}\left(e^{z_{1}-\mathbf{i} z_{2}}-e^{z_{1}+\mathbf{i} z_{2}}\right) \\
& =e^{z_{1}}\left(\frac{1}{2}\left(e^{-i \mathbf{i} z_{2}}+e^{i z_{2}}\right)+\mathbf{j} \frac{\mathbf{i}}{2}\left(e^{-\mathbf{i} z_{2}}-e^{i \mathbf{z} z_{2}}\right)\right) \\
& =e^{z_{1}}\left(\cos \left(z_{2}\right)+\mathbf{j} \sin \left(z_{2}\right)\right) .
\end{aligned}
$$

This concludes our proof.

Clearly, the theorem justifies the following definition. 
Definition 11. We set

$$
e^{Z}:=\lim _{n \rightarrow \infty}\left(1+\frac{Z}{n}\right)^{n}=e^{z_{1}}\left(\cos \left(z_{2}\right)+\mathbf{j} \sin \left(z_{2}\right)\right)
$$

One observes a marvelous similarity with the definitions of the Euler number $e$ and with the exponential functions in real and complex numbers.

We pass now to the properties of this newly introduced bicomplex exponential function.

- First we note that the bicomplex exponential is an extension to $\mathbb{B C}$ of the complex exponential function: indeed, for $Z=z_{1}+\mathbf{j} 0 \in \mathbb{C}$, we have that

$$
e^{Z}=e^{z_{1}}(\cos (0)+\mathbf{j} \sin (0))=e^{z_{1}},
$$

which is the usual complex exponential function.

- Note that $\mathrm{e}^{z_{1}}$ is the complex modulus of the bicomplex number $e^{\mathrm{z}}$, and $z_{2}$ is the complex argument of the same bicomplex number $e^{z}$. The reader may find it instructive to compare this fact with what happens in the complex case.

- For $\mathbf{Z}=\mathbf{0}=0 \mathbf{e}+0 \mathbf{e}^{\dagger}$, we have: $\mathbf{e}^{0}=1 \mathbf{e}+1 \mathbf{e}^{\dagger}=1$.

- For any bicomplex number $\mathrm{Z}$, the exponential $e^{\mathrm{Z}}$ is invertible. This is because

$$
e^{Z}=e^{z_{1}-\mathbf{i} z_{2}} \mathbf{e}+e^{z_{1}+\mathbf{i} z_{2}} \mathbf{e}^{\dagger}
$$

and the exponential terms $e^{z_{1}-\mathbf{i} z_{2}}$ and $e^{z_{1}+\mathbf{i} z_{2}}$ are complex exponential functions, so they are never zero. The inverse multiplicative of $e^{z}$ is

$$
e^{-Z}=e^{-\left(z_{1}-\mathbf{i} z_{2}\right)} \mathbf{e}+e^{-\left(z_{1}+\mathbf{i} z_{2}\right)} \mathbf{e}^{\dagger}=e^{-z_{1}}\left(\cos \left(z_{2}\right)-\mathbf{j} \sin \left(z_{2}\right)\right) .
$$

Thus, the range of the bicomplex exponential function does not contain neither the zero nor any zero divisors.

- Two curious facts. For $\mathbf{e}=1 \cdot \mathbf{e}+0 \cdot \mathbf{e}^{\dagger}$, and $\mathbf{e}^{\dagger}=0 \cdot \mathbf{e}+1 \cdot \mathbf{e}^{\dagger}$, we have:

$$
\begin{aligned}
e^{\mathbf{e}} & =e \cdot \mathbf{e}+1 \cdot \mathbf{e}^{\dagger}=e^{\frac{1}{2}}\left(\cos \left(\frac{\mathbf{i}}{2}\right)+\mathbf{j} \sin \left(\frac{\mathbf{i}}{2}\right)\right) \\
& =e^{\frac{1}{2}}\left(\cosh \left(\frac{1}{2}\right)+\mathbf{j i} \sinh \left(\frac{1}{2}\right)\right) .
\end{aligned}
$$

Similarly:

$$
\begin{aligned}
e^{\mathbf{e}^{\dagger}} & =1 \cdot \mathbf{e}+e \cdot \mathbf{e}^{\dagger}=e^{\frac{1}{2}}\left(\cos \left(\frac{\mathbf{i}}{2}\right)-\mathbf{j} \sin \left(\frac{\mathbf{i}}{2}\right)\right) \\
& =e^{\frac{1}{2}}\left(\cosh \left(\frac{1}{2}\right)-\mathbf{j i} \sinh \left(\frac{1}{2}\right)\right) .
\end{aligned}
$$


- Due to the commutativity of the multiplication in $\mathbb{B C}$, we can show that for any $Z_{1}=$ $z_{11}+\mathbf{j} z_{12}$ and $Z_{2}=z_{21}+\mathbf{j} z_{22}$ in $\mathbb{B C}$, the following formula holds:

$$
e^{Z_{1}} e^{Z_{2}}=e^{Z_{1}+Z_{2}}
$$

Indeed, we have:

$$
\begin{aligned}
e^{z_{1}} e^{z_{2}}= & \left(e^{z_{11}}\left(\cos \left(z_{12}\right)+\mathbf{j} \sin \left(z_{12}\right)\right)\right)\left(e^{z_{21}}\left(\cos \left(z_{22}\right)+\mathbf{j} \sin \left(z_{22}\right)\right)\right) \\
= & e^{z_{11}} e^{z_{21}}\left(\left(\cos \left(z_{12}\right) \cos \left(z_{22}\right)-\sin \left(z_{12}\right) \sin \left(z_{22}\right)\right)\right. \\
& \left.\quad+\mathbf{j}\left(\sin \left(z_{12}\right) \cos \left(z_{22}\right)+\sin \left(z_{22}\right) \cos \left(z_{12}\right)\right)\right) \\
= & e^{z_{11}+z_{21}}\left(\cos \left(z_{12}+z_{22}\right)+\mathbf{j} \sin \left(z_{12}+z_{22}\right)\right)=e^{z_{1}+z_{2}}
\end{aligned}
$$

This equality means that the exponential function is a homomorphism from the additive group of bicomplex numbers into the multiplicative group of invertible bicomplex numbers.

- In the case $\mathbf{Z}=0+\mathbf{j} z_{2}$, we have:

$$
\mathrm{e}^{\mathrm{Z}}=\mathrm{e}^{\mathrm{j} z_{2}}=\cos \left(z_{2}\right)+\mathbf{j} \sin \left(z_{2}\right)
$$

- The complex formula $e^{\mathbf{i} \pi}+1=0$ remains valid for bicomplex numbers, but it is complemented with its mirror image $\mathrm{e}^{\mathrm{j} \pi}+1=0$.

- For any $\mathbf{Z}=\alpha \mathbf{e}+\beta \mathbf{e}^{\dagger} \in \mathbb{B C}$, and any invertible bicomplex number $W=\gamma \mathbf{e}+\delta \mathbf{e}^{\dagger}$, i.e. $\gamma \delta \neq 0$, the equation $e^{Z}=W$ is equivalent to the system $e^{\alpha}=\gamma$ and $e^{\beta}=\delta$. Because $\gamma \delta \neq 0$, it follows that there is always a solution.

- We leave as an exercise to the reader to verify that the bicomplex derivative of $e^{z}$ is still $e^{z}$.

- Recalling that the complex exponential function and the complex trigonometric functions are periodic, we obtain that

$$
\begin{aligned}
e^{Z} & =\mathrm{e}^{z_{1}}\left(\cos \left(z_{2}\right)+\mathbf{j} \sin \left(z_{2}\right)\right) \\
& =\mathrm{e}^{z_{1}+2 \pi \mathbf{m} m}\left(\cos \left(z_{2}+2 \pi \mathfrak{n}\right)+\mathbf{j} \sin \left(z_{2}+2 \pi \mathfrak{n}\right)\right) \\
& =e^{Z+2 \pi(m \mathbf{i}+n \mathbf{j})},
\end{aligned}
$$

for $m$ and $n$ integer numbers. Thus the bicomplex exponential function is periodic with bicomplex periods $2 \pi(m \mathbf{i}+n \mathbf{j})$. One can prove that these are the only periods.

\section{Trigonometric functions of a bicomplex variable}

Adding and subtracting the formulas $e^{\mathbf{j} z_{2}}=\cos \left(z_{2}\right)+\mathbf{j} \sin \left(z_{2}\right)$ and $e^{-\mathbf{j} z_{2}}=\cos \left(z_{2}\right)-\mathbf{j} \sin \left(z_{2}\right)$, for any $z_{2} \in \mathbb{C}$, we express the complex cosine and sine via the bicomplex exponential: 


$$
\begin{aligned}
& \cos z_{2}=\frac{e^{\mathbf{j} z_{2}}+e^{-\mathbf{j} z_{2}}}{2}, \\
& \sin z_{2}=\frac{e^{\mathbf{j} z_{2}}-e^{-\mathbf{j} z_{2}}}{2 \mathbf{j}} .
\end{aligned}
$$

Thus we are in a position to introduce the bicomplex sine and cosine functions which are direct extensions of their complex antecedents.

Definition 12. Let $\mathbf{Z}=z_{1}+\mathbf{j} z_{2} \in \mathbb{B} \mathbb{C}$. We define the bicomplex cosine and sine functions of a bicomplex variable as follows:

$$
\begin{aligned}
& \cos Z:=\frac{e^{j Z}+e^{-j Z}}{2}, \\
& \sin Z:=\frac{e^{j Z}-e^{-j Z}}{2 j} .
\end{aligned}
$$

Given $\mathbf{Z}=z_{1}+\mathbf{j} z_{2}=\alpha \mathbf{e}+\beta \mathbf{e}^{\dagger} \in \mathbb{B} \mathbb{C}$, the properties of the bicomplex exponential bring us immediately to the idempotent representation of $\cos Z$ and $\sin Z$ :

$$
\begin{aligned}
& \cos Z=\cos (\alpha) \mathbf{e}+\cos (\beta) \mathbf{e}^{\dagger} \\
& \sin Z=\sin (\alpha) \mathbf{e}+\sin (\beta) \mathbf{e}^{\dagger}
\end{aligned}
$$

In terms of the components of the cartesian representation, one gets:

$$
\cos \mathbf{Z}=\cos \left(z_{1}-\mathbf{i} z_{2}\right) \mathbf{e}+\cos \left(z_{1}+\mathbf{i} z_{2}\right) \mathbf{e}^{\dagger} .
$$

Since for a complex variable $z$ the following formulas hold:

$$
\cosh (z)=\cos (\mathbf{i} z), \quad \sinh (z)=-\mathbf{i} \sin (\mathbf{i} z)
$$

we obtain that

$$
\cos \mathbf{Z}=\cosh \left(z_{2}\right) \cos \left(z_{1}\right)-\mathbf{j} \sinh \left(z_{2}\right) \sin \left(z_{1}\right)
$$

We continue with a description of some basic properties of the trigonometric bicomplex functions.

- Since the complex sine and cosine functions are periodic with principal period $2 \pi$, then taking $\mathbf{Z}=\alpha \mathbf{e}+\beta \mathbf{e}^{\dagger}$ and setting $Z_{k, \ell}=(\alpha+2 k \pi) \mathbf{e}+(\beta+2 \ell \pi) \mathbf{e}^{\dagger}$ for arbitrary integers $k, \ell$ we have:

$$
\cos \left(Z_{k, \ell}\right)=\cos (Z), \quad \sin \left(Z_{k, \ell}\right)=\sin (Z) .
$$

Thus the real number $(2 \pi) \mathbf{e}+(2 \pi) \mathbf{e}^{\dagger}=2 \pi$ remains the principal period of both bicomplex sine and cosine functions. 
- From (6.3), the equation $\cos Z=0$ is equivalent to the equations in complex variables $\alpha$ and $\beta$ :

$$
\cos (\alpha)=0, \quad \cos (\beta)=0 .
$$

The solutions are $\alpha=\frac{\pi}{2}+k \pi$, and $\beta=\frac{\pi}{2}+\ell \pi$, for $k, \ell \in \mathbb{Z}$. Note that $\alpha$ and $\beta$ are never 0 , so the bicomplex solutions $Z$ to $\cos Z=0$ are always invertible. In the $\{\mathbf{1}, \mathbf{j}\}$ basis, we get the general solution to $\cos Z=0$ as

$$
\mathrm{Z}=z_{1}+\mathbf{j} z_{2}=((1+k+\ell)+\mathbf{j} \mathbf{i}(k-\ell)) \frac{\pi}{2} .
$$

- Similarly, the equation $\sin Z=0$ is equivalent to

$$
\sin (\alpha)=0, \quad \sin (\beta)=0 .
$$

The solutions are $\alpha=k \pi$, and $\beta=\ell \pi$, for $k, l \in \mathbb{Z}$. Note that there are non-invertible solutions for $\sin Z=0$, e.g. for $\alpha=0$, i.e. $k=0$, and $\beta \neq 0$. In the $\{\mathbf{1}, \mathbf{j}\}$ basis, we get the general solution for $\sin Z=0$ as

$$
\mathrm{Z}=z_{1}+\mathbf{j} z_{2}=(k+\ell+\mathbf{j} \mathbf{i}(k-\ell)) \frac{\pi}{2} .
$$

- The component-wise formulas (6.3) guarantee that the usual trigonometric identities are true, e.g., the sums and differences of angle formulas, the double angle identities, etc. For example:

$$
\sin ^{2} Z+\cos ^{2} Z=\left(\sin ^{2}(\alpha)+\cos ^{2}(\alpha)\right) \mathbf{e}+\left(\sin ^{2}(\beta)+\cos ^{2}(\beta)\right) \mathbf{e}^{\dagger}=1 .
$$

- It turns out that both functions have the derivatives which extend directly their complex antecedents, i.e.

$$
\begin{aligned}
(\cos Z)^{\prime} & =-\sin Z, \\
(\sin Z)^{\prime} & =\cos Z .
\end{aligned}
$$

\section{$7 \quad$ Bicomplex Radicals}

In this and the next section we begin the study of inverse functions in $\mathbb{B C}$. We start by looking at the equation $Z^{n}=W$, where $Z=z_{1}+\mathbf{j} z_{2}=\alpha \mathbf{e}+\beta \mathbf{e}^{\dagger}$, and $W=w_{1}+\mathbf{j} w_{2}=\mathbf{a} \mathbf{e}+\mathbf{b e}^{\dagger}$. This system is equivalent to the following two complex equations in variables $\alpha$ and $\beta$ :

$$
\alpha^{n}=a, \quad \beta^{n}=b .
$$

If $W$ is invertible, i.e. $a b \neq 0$, each complex equation has $n$ distinct complex solutions, and the equations are independent of each other. Denote these solutions by $a_{k} \in \sqrt[n]{a}$ and $b_{\ell} \in \sqrt[n]{b}$, respectively. Therefore the bicomplex equation $Z^{n}=W$ has, in general, $n^{2}$ solutions given by the bicomplex numbers

$$
Z_{k \ell}=a_{k} \mathbf{e}+b_{\ell} e^{\dagger}=\frac{a_{k}+b_{\ell}}{2}+j \frac{b_{\ell}-a_{k}}{2 i}
$$


for all $k, \ell=1 \ldots n$. We define the $n$-th root of $W$ to be the set of all of these solutions, $\sqrt[n]{W}:=$ $\left\{Z_{k \ell}\right\}$.

Note that if we start with formula (2.1) for the bicomplex number $W=w_{1}+\mathbf{j} w_{2}$, i.e.

$$
W=|W|_{c}(\cos \theta+\mathbf{j} \sin \theta)
$$

where $|W|_{c}=\sqrt{w_{1}^{2}+w_{2}^{2}}$ is the complex modulus of $W$, and $\theta$ is the complex argument of $W$, then the solutions $Z_{k \ell}$ of the equation $Z^{n}=W$ have complex modulus $\sqrt[n]{|W|_{c}}$, which is a set of $n$ complex numbers, and arguments $\frac{\theta+2 \ell \pi}{n}$, for $\ell=1 \ldots n$. In conclusion, we find again that there are $n^{2}$ bicomplex $n-$ th roots, and more precisely

$$
\sqrt[n]{W}=\left\{\sqrt[n]{|W|_{c}}\left(\cos \frac{\theta+2 \ell \pi}{n}+j \sin \frac{\theta+2 \ell \pi}{n}\right): \ell \in\{0,1, \ldots, n-1\}\right\}
$$

If $\mathbf{W}=\mathbf{a} \mathbf{e}+\mathrm{be}^{\dagger}$ is a zero divisor then exactly one of the complex numbers $\mathrm{a}$ or $\mathrm{b}$ is zero, so the bicomplex equation $Z^{n}=W$ has exactly $n$ solutions, all of them zero divisors. Obviously if $W=0$ there is only one solution, $Z=0$, to the equation $Z^{n}=0$.

\section{The bicomplex logarithmic function}

In this section we define the notion of the logarithm of a bicomplex number. In the complex case, we look for the solutions of $e^{z}=w$, where $z$ and $w \neq 0$ are complex numbers. If $\ln (|w|)$ is the real $\operatorname{logarithm}$ of the positive number $|w|$, and $\arg (w)$ is the principal argument of $w$, then the complex logarithm is defined as the set

$$
\log (w):=\{\ln |w|+\mathbf{i}(\arg (w)+2 m \pi): m \in \mathbb{Z}\}
$$

and its $\mathrm{m}-$ th branch is defined by

$$
\log _{m}(w):=\ln |w|+\mathbf{i}(\arg (w)+2 m \pi) .
$$

We will finally denote by $\log (w)$ the principal branch of $\log (w)$, i.e. the branch for $m=0$. Similarly, we will denote by $\arg (w)$ the principal argument, so that

$$
\operatorname{Arg}(w):=\{\arg (w)+2 m \pi \mid m \in \mathbb{Z}\} .
$$

We note, in this respect, that our notation differs a bit from other more frequently used, but we believe our convention will be useful in discussing the bicomplex case.

We pass now to our task to define the logarithm of a bicomplex number. Take a bicomplex number $Z=z_{1}+\mathbf{j} z_{2}$, and an invertible bicomplex number $\mathbf{W}=w_{1}+\mathbf{j} w_{2}$. We study the solutions to the bicomplex equation $e^{Z}=W$. Recall again from (2.1) that

$$
W=|W|_{\mathfrak{c}}(\cos \theta+\mathbf{j} \sin \theta) .
$$


Then

$$
e^{z_{1}}=|W|_{c}, \quad z_{2}=\theta+2 k \pi
$$

for any $k \in \mathbb{Z}$. The first equation implies that $z_{1}$ is in the set $\log |W|_{c}$, i.e. for every $m \in$ $\mathbb{Z}$ the complex number $z_{1}=\log _{\mathrm{m}}|W|_{\mathrm{c}}$, is a solution of the first equation. In the idempotent representation, taking $\gamma=w_{1}-\mathfrak{i} w_{2}$ and $\delta=w_{1}+\mathfrak{i} w_{2}$, then $w_{1}^{2}+w_{2}^{2}=\gamma \delta$. Then the complex modulus of $W$ is $|W|_{c}=\sqrt{w_{1}^{2}+w_{2}^{2}}=\sqrt{\gamma \delta}$, and

$$
\log _{m}|W|_{c}=\log _{m} \sqrt{\gamma \delta}
$$

One can show that

$$
\theta=\mathbf{i} \log _{\ell} \sqrt{\frac{\gamma}{\delta}}
$$

for any $\ell \in \mathbb{Z}$. The (principal) complex $\operatorname{argument} \arg _{c}(W)$ is given by the formula above for $\ell=0$. Finally we obtain

$$
z_{2}=\theta+2 k \pi=\arg _{c}(W)+2 n \pi,
$$

where $n=k+\ell$ is an integer number. Set

$$
\operatorname{Arg}_{c}(W):=\left\{\arg _{c}(W)+2 n \pi \mid n \in \mathbb{Z}\right\}=i \log \sqrt{\frac{\gamma}{\delta}} .
$$

These facts motivate the following definition:

Definition 13. The bicomplex logarithm is defined by:

$$
\log (W):=\log |W|_{c}+\mathbf{j} \operatorname{Arg}_{c}(W),
$$

which is an infinite set of bicomplex numbers. The $(m, n)$-th branch of the bicomplex logarithm of $W$ is given by:

$$
\log _{m, n}(W):=\log _{m}|W|_{c}+\mathbf{j}\left(\arg _{c}(W)+2 n \pi\right)
$$

for $m$ and $n$ integer numbers.

In the idempotent representation $W=\gamma \mathbf{e}+\delta \mathbf{e}^{\dagger}$, it turns out that

$$
\log (W)=\log (\gamma) \mathbf{e}+\log (\delta) \mathbf{e}^{\dagger}
$$

As in the case of the complex logarithm, the formula (8.4) has to be interpreted in the sense that both terms of the right-hand-side represent the infinite sets of complex numbers multiplied by $\mathbf{e}$ and $\mathbf{e}^{\dagger}$, respectively. This formula is obtained immediately by studying the equation $e^{Z}=W$ in its idempotent form. In more detail, if $Z=\alpha \mathbf{e}+\beta \mathbf{e}^{\dagger}$, then $e^{Z}=W$ is equivalent to the two complex equations

$$
e^{\alpha}=\gamma, \quad e^{\beta}=\delta
$$


which have as solutions the complex $\operatorname{logarithms} \log (\gamma)$ and $\log (\delta)$, respectively. In the cartesian basis, formula (8.4) agrees with definition 13

$$
\begin{aligned}
\log (W) & =\frac{1}{2} \log (\gamma \delta)+\frac{1}{2} \mathbf{j i} \log \frac{\gamma}{\delta}=\log \sqrt{\gamma \delta}+\mathbf{j i} \log \sqrt{\frac{\gamma}{\delta}} \\
& =\log |W|_{c}+\mathbf{j} \operatorname{Arg}_{c}(W) .
\end{aligned}
$$

We state below some properties of the bicomplex logarithm.

- The bicomplex logarithm is not defined for zero-divisors, as the bicomplex exponential $W=$ $e^{Z}$ is always invertible.

- If $\mathbf{Z}=z_{1}+\mathbf{j} z_{2}$ is an invertible bicomplex number, if $m, n \in \mathbb{Z}$, then:

$$
\begin{aligned}
e^{\log _{m, n}(Z)} & =e^{\log _{m}|Z|_{c}+\mathbf{j} \arg _{c}(Z)+2 n \pi j}=e^{\log _{m}|Z|_{c}} e^{\mathbf{j} \arg _{c}(Z)} \\
& =|Z|_{c}\left(\cos \left(\arg _{c}(Z)\right)+\mathbf{j} \sin \left(\arg _{c}(Z)\right)\right)=Z
\end{aligned}
$$

- For $\mathrm{Z}=\mathbf{1}=1+\mathbf{j} 0$, we have:

$$
\log _{m, n}(\mathbf{1})=0+2 m \pi \mathbf{i}+2 n \pi \mathbf{j}
$$

for all $m, n \in \mathbb{Z}$.

- For $\mathrm{Z}_{1}$ and $\mathrm{Z}_{2}$ two invertible bicomplex numbers, the following formula holds

$$
\log \left(Z_{1} Z_{2}\right)=\log \left(Z_{1}\right)+\log \left(Z_{2}\right)
$$

The inverses of the bicomplex trigonometric functions are defined in complete analogy with the complex case, as we have already properly defined the notions of bicomplex exponential, logarithm, and square root.

For example, the inverse of the bicomplex cosine function is obtained by solving the equation

$$
\cos (Z)=\frac{e^{\mathbf{j} Z}+e^{-j \mathbf{z}}}{2}=W .
$$

This is a quadratic equation in $\mathrm{e}^{\mathrm{j} Z}$ with roots

$$
e^{\mathrm{j} z}=W \pm \sqrt{W^{2}-1} .
$$

Therefore, for $m, n \in \mathbb{Z}$,

$$
\begin{gathered}
\arccos (W):=-\mathbf{j} \log _{m, n}\left(W \pm \sqrt{W^{2}-1}\right)= \pm \mathbf{j} \log _{m, n}\left(W+\sqrt{W^{2}-1}\right) . \\
\text { Received: May 2011. Revised: October } 2011 .
\end{gathered}
$$




\section{References}

[1] Ahlfors, L., Complex Analysis, McGraw Hill, New York, 1966.

[2] Charak, K.S., Rochon, D., Sharma, N., Normal families of bicomplex holomorphic functions, ArXiv:0806.4403v1 (2008).

[3] Fueter, R., Analytische Funktionen einer Quaternionen Variablen, Comm. Math. Helv. 4 (1932), 9-20.

[4] MoisiL, G., Sur les quaternions monogenes, Bull. Sci. Math. Paris 55 (2) (1931), 169-194.

[5] Moisil, G., Teodorescu N., Fonctions holomorphes dans l'espace, Mathematica (Cluj) 5 (1931), 142-159.

[6] Pogorui, A.A., Rodriguez-Dagnino, R.M., On the set of zeros of bicomplex polynomials, Complex Variables and Elliptic Equations. 51, 7 (2006), 725-730.

[7] Price, G.B., An Introduction to Multicomplex Spaces and Functions, Monographs and Textbooks in Pure and Applied Mathematics, 140, Marcel Dekker, Inc., New York, 1991.

[8] Rochon, D., Shapiro, M., On algebraic properties of bicomplex and hyperbolic numbers, An. Univ. Oradea Fasc. Mat. 11 (2004), 71-110.

[9] Rochon, D., On a relation of bicomplex pseudoanalytic function theory to the complexified stationary SchrÃ $\llbracket$ dinger equation, Complex Var. Elliptic Equ. 53 (2008), 501-521.

[10] Ryan, J., Complexified Clifford analysis, Complex Variables and Elliptic Equations 1 (1982), $119-149$.

[11] Ryan, J., $\mathbb{C}^{2}$ extensions of analytic functions defined in the complex plane, Adv. in Applied Clifford Algebras 11 (2001), 137-145.

[12] Segre, C., Le rappresentazioni reali delle forme complesse e gli enti iperalgebrici, Math. Ann. 40 (1892), 413-467.

[13] Scorza Dragoni, G., Sulle funzioni olomorfe di una variabile bicomplessa, Reale Accad. d'Italia, Mem. Classe Sci. Nat. Fis. Mat. 5 (1934), 597-665.

[14] Spampinato, N., Estensione nel campo bicomplesso di due teoremi, del Levi-Civita e del Severi, per le funzioni olomorfe di due variabili bicomplesse I, II, Reale Accad. Naz. Lincei 22 (1935), 38-43, 96-102.

[15] Spampinato, N., Sulla rappresentazione di funzioni di variabile bicomplessa totalmente derivabili, Ann. Mat. Pura Appli. 14 (1936), 305-325. 